\title{
Review
}

\section{How Do Bacteria Maximize Their Cellular Assets?}

\author{
Juhyun Kim* \\ School of Life Science, BK21 FOUR KNU Creative BioResearch Group, Kyungpook National University, Daegu 41566, Republic of Korea
}

Received: October 27, 2021 / Revised: December 13, 2021 / Accepted: December 15, 2021

Cellular resources including transcriptional and translational machineries in bacteria are limited, yet microorganisms depend upon them to maximize cellular fitness. Bacteria have evolved strategies for using resources economically. Regulatory networks for the gene expression system enable the cell to synthesize proteins only when necessary. At the same time, regulatory interactions enable the cell to limit losses when the system cannot make a cellular profit due to fake substrates. Also, the architecture of the gene expression flow can be advantageous for clustering functionally related products, thus resulting in effective interactions among molecules. In addition, cellular systems modulate the investment of proteomes, depending upon nutrient qualities, and fast-growing cells spend more resources on the synthesis of ribosomes, whereas nonribosomal proteins are synthesized in nutrient-limited conditions. A deeper understanding of cellular mechanisms underlying the optimal allocation of cellular resources can be used for biotechnological purposes, such as designing complex genetic circuits and constructing microbial cell factories.

Keywords: Resource allocation, growth law, regulatory network, cellular economy

\section{Introduction}

When given a choice between identical goods, no one would purchase costlier commodities. Instead, a person would select the cheapest option, as it would provide us with the best value. This choice is a result of economic behavior that manages scarce resources optimally to maximize desire. In addition, such a rational choice allows us to survive and prosper in a competitive environment.

By the same token, bacteria are capable of adapting to diverse environmental niches by optimizing the allocation of limited cellular resources. In nutrient-rich conditions, microorganisms can increase ribosome content, thus resulting in a higher ATP budget with larger gene expression machineries [1-3]. Eventually, this contributes to maximizing cellular fitness. On the other hand,

\section{*Corresponding author}

Tel.: +82-53-950-5379

E-mail: juhyunkim@knu.ac.kr when cells grow in a nutrient-poor medium, a minimum fraction of cellular resources is allocated to maintain a certain level of growth [4, 5]. This linear relationship between the mass fraction of cellular components and the growth rate indicates that bacteria control the use of their cellular assets, which represent energy currency and building blocks [5]. In other words, a bacterial cell can be considered as a closed economic system [6]. Because of the cellular complexity, it is not straightforward to engineer a biological system with synthetic circuits in vivo for producing recombinant proteins. To predict and engineer rational biological systems, it is necessary to understand how cells manage limited cellular resources optimally.

Biological systems are composed of dynamic networks that determine the gene expression level depending upon both intracellular and extracellular cues [7-9]. Owing to the regulatory network of the gene expression system, living organisms can synthesize only necessary proteins in given conditions. This allows cells to reduce the protein synthesis cost, which is defined as a reduced 
growth rate due to limited cellular resources with a lower gene expression capacity $[10,11]$. At the same time, the cost and benefits of protein expression are also well-adjusted in evolution. A previous study has shown that half of the Escherichia coli proteome is not used in a given condition [12]. As a result of the expression of unnecessary proteins, the growth rate decreases; however, this bet-hedging strategy can be potentially useful under changing environments [13]. For instance, a clonal $E$. coli population splits into growing and nongrowing persister phenotypes after glucose-gluconeogenic substrate shifts. While cells that grew fast on glucose achieved a high gluconeogenic flux, the subpopulation that was refrained from growth showed higher antibiotic tolerance [14]. Bet-hedging is thus linked to survival under conditions in which the specialization of parts of the population can benefit the whole group. This means that cells maximize their probability of survival and reproduction by maintaining a certain level of phenotypic diversity in a population at the expense of reducing the gene expression capacity. The emergence of nongenetic phenotypic heterogeneity is the result of the stochasticity of gene expression, also known as "noise" in gene expression. This nongenetic phenotypic heterogeneity influences cell-to-cell phenotypic variations in populations, and the stochasticity results from the low availability of cellular components associated with the gene expression hardware, including synthesis of RNA and proteins [15, 16]. Accordingly, cells need to exploit their resources economically with respect to the tradeoff between resource availability and growth.

With respect to the gene expression system in a bacterial cell, this review addresses current understanding of cellular strategies for managing scarce resources and for optimizing cellular fitness with respect to the gene expression system. Moreover, the description below provides a guide for manipulating resource allocation for diverse biotechnological purposes.

\section{Operons and Their Regulatory Architec- ture Confer Several Benefits}

Many functionally related genes are grouped into operons and are coregulated by particular cues in bacteria. As a result of regulation, a single polycistronic mRNA molecule can be transcribed from cluster genes,
A

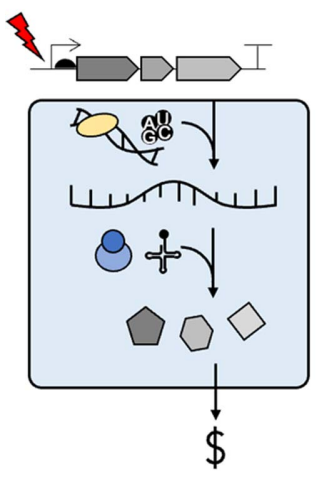

Cellular Assets

=Energy currency and building blocks
B

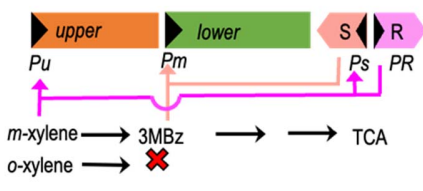

C

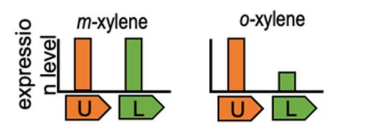

D

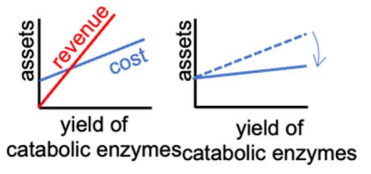

Fig. 1. Production process of cellular assets. (A) Bacterial cells obtain energy and building blocks as revenues through catabolic enzymes by spending protein synthesis costs. (B) The regulatory network of the TOL system in P. putida mt- 2 . $m$-xylene can be transformed into 3-methylbenzoate $(3 \mathrm{MBz})$ using the upper operon, and the metabolite is further catabolized by the lower operon and TCA cycle. Although o-xylene is partially cleaved through the upper operon, it produces a dead-end metabolite. (C) Relative expression profile of the TOL system responding to $m$-xylene or $o$-xylene [29]. (D) $m$-xylene enables cells to yield assets, whereas 0 -xylene does not provide any revenue. However, cells can limit losses due to the reduced activity of the lower operon.

which are governed by the same expression system. Due to spatial organization of genes and an efficient mechanism for activating/inhibiting grouped genes, cells can achieve economic allocation of cellular resources. In turn, synthesis of the right enzymes at the right time as the investment step is a way to produce more cellular assets, which appear as energy sources and building blocks (Fig. 1A).

The best examples of these strategies are well addressed in $E$. coli with lactose metabolism and tryptophan biosynthesis [17-19]. In the presence of lactose, the promoter of the lac operon consisting of lacZ, lacY, and lacA genes can be stimulated, which switches all of these genes on. Also, Trp repressor prevents the synthesis of enzymes responsible for tryptophan biosynthesis by inhibiting the Trp operon when tryptophan is sufficient; only when the cellular concentration of the amino acid falls below a certain level does the repressor returns to its inactive form, and the polycistronic Trp mRNAs are transcribed [20]. These regulatory mechanisms indicate that built-in programming enables cellular resources to 
be spent conditionally for the synthesis of specific RNAs/ enzymes, and the switchable operation leads to the production of larger cellular assets.

However, such an expression system achieves no return on investment. This failure occurs when non-productive substrates enhance the switch of metabolic systems. Although bacterial transcriptional factors are robust and evolvable, they sense fake chemicals that do not provide any returns. This aspect is observed in the TOL system, which is encoded by the plasmid pWW0 of Pseudomonas putida mt-2. This soil bacterium is capable of metabolizing $m$-xylene and other related aromatics by using a set of enzymes encoded by "upper" and "lower" plasmid-borne operons [21-23]. Although all the responsible genes are co-transcribed in two different polycistronic mRNAs, the transcriptional networks are well-coordinated to activate both operons. For instance, in the presence of $m$-xylene, XylR, which is the main regulator of the TOL system, activates both the $P u$ promoter (responsible for the upper pathway operon) and the Ps promoter, which stimulates the expression of the $x y l S$ gene. As a result, the XylS protein induces the lower operon by activating the Pm promoter with its effector 3-methylbenzoate (3MBz), an intermediate metabolite of $m$-xylene biodegradation [24-27] (Fig. 1B). Accordingly, the TOL system delivers revenues with the aromatic compound through the two pathways, and the profit can be considered the difference between the revenue and the cost [28]. Given the regulatory network of the TOL system, once the upper operon is activated, the lower pathway is autonomously triggered [29]. A key regulator of the TOL system, XylR can sense diverse aromatic compounds, including toluene and $o$-xylene [29-31], although the latter compound is a non-energy producing substrate (Fig. 1C). However, this does not mean that the fake substrate collapses the resource management system in the cell. Instead, cells cope with the risk and reduce loss by minimizing the synthesis costs of catabolic enzymes. It was previously reported that the expression level of the lower pathway decreased in response to $o$-xylene [29], which can result in a reduced operation cost of the TOL system [28] (Fig. 1D). By the same token, the profit increases by expressing only the lower operon to metabolize 3MBz. Accordingly, cells can not only manage profit maximization but also limit losses by taking advantage of non-continuous operon structures and regulatory networks.

\section{Organization of Macromolecules at the Right Position is Advantageous for Carry- ing out Various Functions Effectively}

Spatial organization of macromolecules can provide an extra layer for optimizing the use of resources, because the physical arrangements of clustering of functionally related molecules in a system generate efficient interactions [32]. For instance, the expression flow of the $x y l$ genes of the TOL catabolic system is spatially organized in P. putida [33, 34]. On the basis of spatial localization of $x y l$ transcripts with respect to cellular components, including host chromosomal DNA, TOL plasmid, and gene expression hardware, such as RNAP and ribosomes, it was demonstrated that the mRNAs were neighbored but were not present on the TOL plasmid and cellular nucleoid (Fig. 2A) [33]. In addition, the transcripts were majorly concentrated on ribosome-rich regions of the cell $[33,34]$. The spatial organization of macromolecules suggested that $x y l$ mRNAs migrate from their transcriptional sites to ribosomes for translation [33]. On the other hand, the architecture of gene expression flow can be advantageous for the assembly of cognate catabolic enzymes encoded by TOL operons for the degradation of responsible aromatic compounds (Fig. $2 \mathrm{~A})$. This is because catabolic enzymes synthesized in the cellular structure remain close to each other, which
A

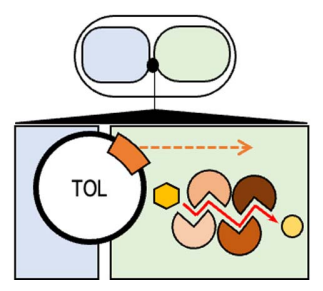

B

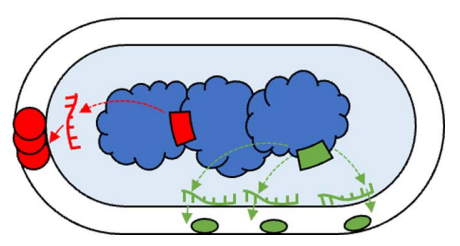

Fig. 2. Spatial organization of cellular components. (A) Gene expression machinery, such as RNAP and ribosomes, are compartmentalized. The TOL plasmid of $P$. putida resides close to transcriptional regions (blue), whereas its transcripts migrate to ribosome-rich sites (green); the products can be easily clustered. This clustering can generate metabolic channeling, which accelerates enzyme catalysis activities. (B) Proteins localize at their functional cellular domains through RNA locomotion mechanisms using signal receptor proteins and Zip-code sequences. 
enables the formation of metabolic channeling, thus accelerating catabolic activities [35].

Interestingly, many proteins localize at their functional cellular domains through RNA targeting. It was reported that Bacillus subtilis positioned ComE proteins at septal and polar regions to develop efficient competence of the cell [36]. Interestingly, com $E$ mRNA accumulated in the same subcellular region, where its coding protein resided. It was revealed that this spatial distribution was dependent on transacting factors, such as DivIVA and ComN, which are involved in cell pole differentiation and the regulation of competence gene expression, respectively [36]. Also, polar localization of the flaA mRNA, encoding the major flagellin, was detected in the elongation of Campylobacter jejuni [37]. In addition to such exemplary proteins, proteome localization is corelated with RNA distribution at the genome-wide level (Fig. 2B). Both approaches utilizing fluorescent in situ hybridization with complex probe sets [38] and RNA localization by fractionation and sequencing [39] revealed that most mRNAs encoding membrane proteins were preferentially positioned at the membrane. Moreover, mRNAs encoding cytoplasmic and polar proteins were largely distributed throughout these respective cellular regions [40]. It is known that the cis-acting sequence in the transcript serves as a ZIP code [41] or signal recognition particle [38] that can determine protein targeting in a particular region through RNA localization. Although the molecular mechanisms of protein localization have yet to be investigated, the spatial distribution of macromolecules to their operative positions enables cells to carry out functions effectively.

\section{Modulation of Proteome Partitioning is Cost Effective for Protein Synthesis}

Gene expression machineries including RNA polymerase and ribosomes are not abundant in bacterial cells, and therefore, the expression of a particular gene can affect the activity of another seemingly unconnected gene [42-44]. This is mainly caused by the limited number of ribosomes and growing number of observations that have shown that the accumulation of one gene product led to a decrease in the expression of the other gene in a circuit following a linear relationship [42-48]. This tradeoff also appears with cellular proteomes. In E. coli,

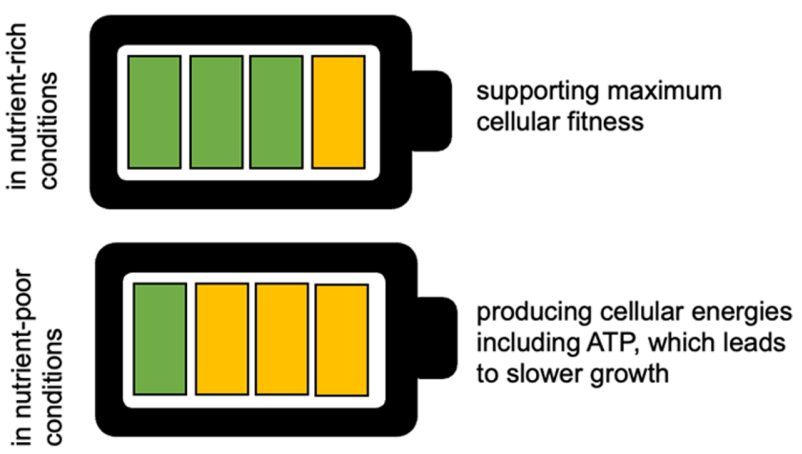

Fig. 3. Tuning the allocation of cellular resources. Bacteria can modulate the investment of cellular proteomes depending on nutrient qualities. More number of proteomes are allocated to ribosome-associated proteins (green boxes) under nutrientrich conditions to maximize cellular fitness. In contrast, cells synthesize more metabolic proteins (yellow boxes) to obtain energy at the expense of ribosome production under poor nutrient conditions.

more of the available cellular proteomes are allocated to ribosome-associated fractions, as they grow fast in nutrient-rich media [3, 49, 50]. However, cells needs to make more metabolic proteins to earn cellular assets at the expense of ribosome synthesis under a nutrient-limited condition, thus resulting in slower growth $[3,49,50]$. On this basis, the partition of cellular proteomes is linked with ribosome synthesis and the production of nonribosomal proteins, and conditional distribution of cellular proteome enables cells to allocate resources cost-effectively, as the synthesis of ribosomes is energetically costly [51, 52] (Fig. 3). How do they achieve an optimal concentration of ribosomes, and what is the underlying molecular mechanism?

The key source of the translational machineries is $r r n$ operons in bacteria, and E. coli carries seven copies of the operon. Interestingly, an isogenic strain with higher rrn operon numbers (9 and 10) showed a slower growth rate as well as longer lag phase time, and the same growth profile appeared in strains carrying either 5 or 6 $r r n$ operon numbers in fluctuating, nutrient-rich conditions [53]. These results suggest that evolutionary tradeoffs in wild-type $E$. coli leads to a set optimal rRNA operon number. Also, the ideal concentration of ribosomes can be controlled by the intracellular alarmone [5458]. When amino acids or other nutrients are limited, cells accumulate the alarmone guanosine tetra- or penta-phosphate (p)ppGpp, which is synthesized by 
RelA and/ or SpoT proteins [54-58]. The alarmone then inhibits the transcription of both rRNAs and ribosomalprotein coding genes through direct interaction with RNA polymerase [59]. In contrast, the concentration of the (p)ppGpp decreases in modest growth conditions with more favorable nutrients.

Remarkably, the translation elongation rate also contributes to the cellular economy. It was recently reported that glycerol-grown cells exhibited lower gene expression capacity compared to that in glucose, although the same growth rate was set for both culture conditions [5]. Given that glycerol does not provide equal quality of translational substrates, including amino acids relative to glucose, the computational approach reasoned that the mass fraction of ribosomes rises in resource production to maintain the growth rate, thus resulting in a lower elongation rate [5]. This observation strongly demonstrates that a subtle interaction between the elongation rate and ribosome concentration contributes cells to exploit cellular resources, depending upon their environments.

\section{Conclusion}

The transfer of genetic information plays a fundamental role in living organisms, and this is mainly achieved by the gene expression system. Maintaining and operating the system economically is crucial for maximizing cellular fitness. In this respect, bacteria possess several strategies for the optimal allocation of cellular DNA organization, and operon structures with regulatory networks enable the cell to reduce its operating costs for synthesizing proteins; thus, they can achieve either higher production or minimized losses in response to energy producing substrates or fake substrates, respectively. In addition, spatial distribution of cellular components into functional domains contributes to efficient and effective interactions among functionally related molecules. Moreover, the cell is capable of modulating the partition of cellular proteomes, depending upon nutrient quality; therefore the cellular budget is used economically. These findings guide better understanding of such strategies with respect to the gene expression system, which enables us to design rational DNA circuits, pathways, and a cellular chassis that support the maximum expression of desired biologics. Furthermore, manipulation of the allocation resource is crucial for engineering living systems for advanced bacterial programming.

\section{Conflict of Interests}

The authors have no financial conflicts of interest to declare.

\section{References}

1. Klumpp S, Scott M, Pedersen S, Hwa T. 2013. Molecular crowding limits translation and cell growth. Proc. Natl. Acad. Sci. USA 110: 16754-16759.

2. Klumpp S, Zhang Z, Hwa T. 2009. Growth rate-dependent global effects on gene expression in bacteria. Cell 139: 1366-1375.

3. Scott M, Klumpp S, Mateescu EM, Hwa T. 2014. Emergence of robust growth laws from optimal regulation of ribosome synthesis. Mol. Syst. Biol. 10: 747.

4. Korem Kohanim $Y$, Levi D, Jona G, Towbin BD, Bren A, Alon U. 2018. A bacterial growth law out of steady state. Cell Rep. 23: 2891-2900.

5. Kim J, Darlington APS, Bates DG, Jimenez Jl. 2021. The interplay between growth rate and nutrient quality defines gene expression capacity. bioRxiv. 2021.2004.2002.438188.

6. Kim J, Darlington A, Salvador M, Utrilla J, Jimenez J. 2019. Trade-offs between gene expression, growth and phenotypic diversity in microbial populations. Curr. Opin. Biotechnol. 62: 29-37.

7. Sneppen K, Krishna S, Semsey S. 2010. Simplified models of biological networks. Annu. Rev. Biophys. 39: 43-59.

8. Lozada-Chavez I, Janga SC, Collado-Vides J. 2006. Bacterial regulatory networks are extremely flexible in evolution. Nucleic Acids Res. 34: 3434-3445.

9. Shis DL, Matthew R, Bennett, Igoshin OA. 2018. Dynamics of bacterial gene regulatory networks. Ann. Rev. Biophys. 47: 447-467.

10. Vind J, Sorensen MA, Rasmussen MD, Pedersen S. 1993. Synthesis of proteins in Escherichia coli is limited by the concentration of free ribosomes. Expression from reporter genes does not always reflect functional mRNA levels. J. Mol. Biol. 231: 678-688.

11. Shachrai I, Zaslaver A, Alon U, Dekel E. 2010. Cost of unneeded proteins in $E$. coli is reduced after several generations in exponential growth. Mol. Cell 38: 758-767.

12. O'Brien EJ, Utrilla J, Palsson BO. 2016. Quantification and classification of E. coli proteome utilization and unused protein costs across environments. PLoS Comput. Biol. 12: e1004998.

13. Mori M, Schink S, Erickson DW, Gerland U, Hwa T. 2017. Quantifying the benefit of a proteome reserve in fluctuating environments. Nat. Commun. 8: 1225.

14. Kotte O, Volkmer B, Radzikowski JL, Heinemann M. 2014. Phenotypic bistability in Escherichia coli's central carbon metabolism. Mol. Syst. Biol. 10: 736.

15. van Boxtel C, van Heerden JH, Nordholt N, Schmidt P, Bruggeman FJ. 2017. Taking chances and making mistakes: non-genetic phe- 
notypic heterogeneity and its consequences for surviving in dynamic environments. J. R. Soc. Interface 14: 20170241.

16. Martins BM, Locke JC. 2015. Microbial individuality: how singlecell heterogeneity enables population level strategies. Curr. Opin. Microbiol. 24: 104-112.

17. Mackey MC, Santillan M, Yildirim N. 2004. Modeling operon dynamics: the tryptophan and lactose operons as paradigms. $C R$ Biol. 327: 211-224.

18. Lawrence JG. 2002. Shared strategies in gene organization among prokaryotes and eukaryotes. Cell 110: 407-413.

19. Osbourn AE, Field B. 2009. Operons. Cell. Mol. Life Sci : CMLS 66: 3755-3775.

20. Merino E, Jensen RA, Yanofsky C. 2008. Evolution of bacterial trp operons and their regulation. Curr. Opin. Microbiol. 11: 78-86.

21. Assinder SJ, Williams PA. 1990. The TOL plasmids: determinants of the catabolism of toluene and the xylenes. Adv. Microb. Physiol. 31: 1-69.

22. Marques S, Manzanera M, Gonzalez-Perez MM, Ruiz R, Ramos JL. 1999. Biodegradation, plasmid-encoded catabolic pathways, host factors and cell metabolism. Environ. Microbiol. 1: 103-104.

23. Worsey MJ, Williams PA. 1975. Metabolism of toluene and xylenes by Pseudomonas (putida (arvilla) mt-2: evidence for a new function of the TOL plasmid. J. Bacteriol. 124: 7-13.

24. Bertoni G, Marques S, de Lorenzo V. 1998. Activation of the tolueneresponsive regulator $X y / R$ causes a transcriptional switch between sigma54 and sigma70 promoters at the divergent $\mathrm{Pr} / \mathrm{Ps}$ region of the TOL plasmid. Mol. Microbiol. 27: 651-659.

25. Marques S, Gallegos MT, Manzanera M, Holtel A, Timmis KN, Ramos JL. 1998. Activation and repression of transcription at the double tandem divergent promoters for the $x y / R$ and $x y / S$ genes of the TOL plasmid of Pseudomonas putida. J. Bacteriol. 180: 2889-2894.

26. Ramos JL, Marques S, Timmis KN. 1997. Transcriptional control of the Pseudomonas TOL plasmid catabolic operons is achieved through an interplay of host factors and plasmid-encoded regulators. Ann. Rev. Microbiol. 51: 341-373.

27. Gallegos MT, Marques S, Ramos JL. 1996. Expression of the TOL plasmid xylS gene in Pseudomonas putida occurs from a alpha 70-dependent promoter or from alpha 70- and alpha 54dependent tandem promoters according to the compound used for growth. J. Bacteriol. 178: 2356-2361.

28. Kim J, Silva-Rocha R, de Lorenzo V. 2021. Picking the right metaphors for addressing microbial systems: economic theory helps understanding biological complexity. Int. Microbiol. 24: 507519.

29. Kim J, Perez-Pantoja D, Silva-Rocha R, Oliveros JC, de Lorenzo V. 2016. High-resolution analysis of the $\mathrm{m}$-xylene/toluene biodegradation subtranscriptome of Pseudomonas putida mt-2. Environ. Microbiol. 18: 3327-3341.

30. Silva-Rocha Randde Lorenzo V. 2012. Stochasticity of TOL plasmid catabolic promoters sets a bimodal expression regime in Pseudomonas putida mt-2 exposed to m-xylene. Mol. Microbiol. 86: 199-211.
31. Vitale E, Milani A, Renzi F, Galli E, Rescalli E, de Lorenzo V, et al. 2008. Transcriptional wiring of the TOL plasmid regulatory network to its host involves the submission of the sigma54-promoter Pu to the response regulator PprA. Mol. Microbiol. 69: 698713.

32. Zimmerman SB, Trach SO. 1991. Estimation of macromolecule concentrations and excluded volume effects for the cytoplasm of Escherichia coli. J. Mol. Biol. 222: 599-620.

33. Kim J, Goni-Moreno A, de Lorenzo V. 2021. Subcellular architecture of the xyl gene expression flow of the TOL catabolic plasmid of Pseudomonas putida mt-2. mBio. 12: e03685-20.

34. Kim J, Goni-Moreno A, Calles B, de Lorenzo V. 2019. Spatial organization of the gene expression hardware in Pseudomonas putida. Environ. Microbiol. 21: 1645-1658.

35. Castellana M, Wilson MZ, Xu Y, Joshi P, Cristea IM, Rabinowitz JD, et al. 2014. Enzyme clustering accelerates processing of intermediates through metabolic channeling. Nat. Biotechnol. 32: 10111018.

36. dos Santos VT, Bisson-Filho AW, Gueiros-Filho FJ. 2012. DivIVAmediated polar localization of ComN, a posttranscriptional regulator of Bacillus subtilis. J. Bacteriol. 194: 3661-3669.

37. Dugar G, Svensson SL, Bischler T, Waldchen S, Reinhardt R, Sauer $\mathrm{M}$, et al. 2016. The CsrA-FliW network controls polar localization of the dual-function flagellin mRNA in Campylobacter jejuni. Nat. Commun. 7: 11667.

38. Moffitt JR, Pandey S, Boettiger AN, Wang S, Zhuang X. 2016. Spatial organization shapes the turnover of a bacterial transcriptome. Elife 5: e13065.

39. Kannaiah S, Livny J, Amster-Choder O. 2019. Spatiotemporal organization of the $E$. coli transcriptome: Translation independence and engagement in regulation. Mol. Cell 76: 574-589 e577.

40. Fazal FM, Chang HY. 2019. Subcellular spatial transcriptomes: Emerging frontier for understanding gene regulation. Cold Spring Harb Symp. Quant. Biol. 84: 31-45.

41. Nevo-Dinur K, Nussbaum-Shochat A, Ben-Yehuda S, AmsterChoder O. 2011. Translation-independent localization of mRNA in E. coli. Science 331: 1081-1084.

42. Gyorgy A, Jimenez Jl, Yazbek J, Huang HH, Chung H, Weiss R, et al. 2015. Isocost lines describe the cellular economy of genetic circuits. Biophys. J. 109: 639-646.

43. Carbonell-Ballestero M, Garcia-Ramallo E, Montanez R, RodriguezCaso C, Macia J. 2016. Dealing with the genetic load in bacterial synthetic biology circuits: convergences with the Ohm's law. Nucleic Acids Res. 44: 496-507.

44. Shopera T, He L, Oyetunde T, Tang YJ, Moon TS. 2017. Decoupling resource-coupled gene expression in living cells. ACS Synth. Biol. 6: $1596-1604$.

45. Borkowski O, Bricio C, Murgiano M, Rothschild-Mancinelli B, Stan GB, Ellis T. 2018. Cell-free prediction of protein expression costs for growing cells. Nat. Commun. 9: 1457.

46. Ceroni F, Algar R, Stan GB, Ellis T. 2015. Quantifying cellular capacity identifies gene expression designs with reduced burden. Nat. Methods 12: 415-418. 
47. Ceroni F, Boo A, Furini S, Gorochowski TE, Borkowski O, Ladak YN, et al. 2018. Burden-driven feedback control of gene expression. Nat. Methods 15: 387-393.

48. Darlington APS, Kim J, Jimenez Jl, Bates DG. 2018. Engineering translational resource allocation controllers: Mechanistic models, design guidelines, and potential biological implementations. ACS Synth. Biol. 7: 2485-2496.

49. Scott M, Gunderson CW, Mateescu EM, Zhang Z, Hwa T. 2010. Interdependence of cell growth and gene expression: origins and consequences. Science 330: 1099-1102.

50. Scott M, Hwa T. 2011. Bacterial growth laws and their applications. Curr. Opin. Biotechnol. 22: 559-565.

51. Baracchini E, Bremer H. 1991. Control of rRNA synthesis in Escherichia coli at increased rrn gene dosage. Role of guanosine tetraphosphate and ribosome feedback. J. Biol. Chem. 266: 1175311760.

52. Stevenson BS, Schmidt TM. 2004. Life history implications of rRNA gene copy number in Escherichia coli. Appl. Environ. Microbiol. 70: 6670-6677.

53. Gyorfy Z, Draskovits G, Vernyik V, Blattner FF, Gaal T, Posfai G. 2015. Engineered ribosomal RNA operon copy-number variants of $E$. coli reveal the evolutionary trade-offs shaping rRNA operon number. Nucleic Acids Res. 43: 1783-1794.

54. Corrigan RM, Bellows LE, Wood A, Grundling A. 2016. ppGpp negatively impacts ribosome assembly affecting growth and antimicrobial tolerance in Gram-positive bacteria. Proc. Natl. Acad. Sci. USA 113: E1710-1719.

55. Lemke JJ, Sanchez-Vazquez P, Burgos HL, Hedberg G, Ross W, Gourse RL. 2011. Direct regulation of Escherichia coli ribosomal protein promoters by the transcription factors ppGpp and DksA. Proc. Natl. Acad. Sci. USA 108: 5712-5717.

56. Magnusson LU, Farewell A, Nystrom T. 2005. ppGpp: a global regulator in Escherichia coli. Trends Microbiol. 13: 236-242.

57. Sun D, Lee G, Lee JH, Kim HY, Rhee HW, Park SY, et al. 2010. A metazoan ortholog of SpoT hydrolyzes ppGpp and functions in starvation responses. Nat. Struc. Mol. Biol. 17: 1188-1194.

58. Zhu M, Dai X. 2019. Growth suppression by altered (p)ppGpp levels results from non-optimal resource allocation in Escherichia coli. Nucleic Acids Res. 47: 4684-4693.

59. Ross W, Sanchez-Vazquez P, Chen AY, Lee JH, Burgos HL, Gourse RL. 2016. ppGpp binding to a site at the RNAP-DksA interface accounts for its dramatic effects on transcription initiation during the stringent response. Mol. Cell 62: 811-823. 